\title{
Addressing front-line pressures: improvement of patient flow from emergency department streaming to the ambulatory assessment unit at the John Radcliffe Hospital, Oxford University NHS Foundation Trust
}

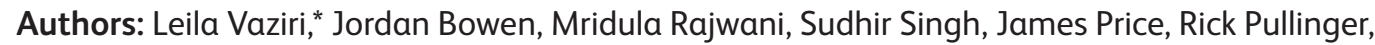 \\ Anuja Bambaravanage* and Nick Parker
}

\section{Background \\ The front line emergency services within the NHS are facing significant pressures, in particular over the winter period. Following the future hospitals report which recommended ambulatory care by default, an ambulatory assessment unit (AAU) was set up at the John Radcliffe Hospital, Oxford in 2016. An NHS Improvement initiative including emergency department (ED) streaming and urgent care (GP) were also added to the ED structure at the trust in December 2017. Initial feedback from the ED streaming team was lack of clarity of suitability of patients for AAU as no pre-existing criteria in place.}

\section{Aims}

To decongest the ED during busy times by increasing the number of direct appropriate referrals from ED streaming to the AAU.

Raising awareness of the ED streaming team and the wider team regarding suitable AAU patients.

\section{Methods}

> Initial observational data collection of patients from ED triage and streaming seen in $A A U$, including mode of referral.

$>$ Identification of barriers to referral.

> Removal of the phone barrier (10 patients via electronic message between 08.00-17.00).

> Poster created of appropriate medical presentations to AAU to have in ED streaming area.

> Post-intervention data collection to review any changes to practice.

\section{Results}

> Pre-intervention, on a single day only $1 / 76(1.3 \%)$ patients seen in ED streaming was referred to AAU. On review, $21 \%$ of the

Authors: Oxford University Hospitals NHS Foundation Trust ${ }^{*} \mathrm{RCP}$ chief registrar patients seen that day in ED streaming would have been AAU suitable.

> Following introduction of the referral checklist and discussion with ED streaming leads and team, a re-review of the ED patients to AAU was performed.

> The AAU ED patient numbers increased from $2.7 \%$ to $14 \%$, and the number of ED streaming referrals went up from $3.3 \%$ to $28 \%$. An additional positive was a significant improvement in the communicated referrals via the electronic system (from $50 \%$ to $73 \%)$.

\section{Conclusion}

The barriers to referral were identified and addressed using simple interventions. These results show a significant improvement in patient flow from ED streaming to AAU between 08.00 and 20.00 . Ongoing work is being planned to address the patient journey and experience in this exercise. 